\title{
Identification of Barriers to Appropriate Dietary Behavior in Low-Income Patients with Type 2 Diabetes Mellitus
}

Todd R. Marcy · Mark L. Britton · Don Harrison

Received: November 8, 2010 / Published online: January 21, 2011

(C) The Author(s) 2011. This article is published with open access at Springerlink.com

\section{ABSTRACT}

Objective: To identify barriers to appropriate dietary behavior in an urban, low-income population of patients with type 2 diabetes and to examine a new instrument in the identification of these barriers in this population. Methods: A cross-sectional survey was developed, validated, and anonymously administered to low-income adults with type 2 diabetes in an academic family medicine physician group practice with a pharmacist-operated diabetes education and comanagement service. The survey consisted of three key subscales: determinants of food selection, importance of life challenges, and barriers to appropriate eating. Results: The survey was administered to 98 patients with a mean age of 51.98 years, a mean duration of diabetes of 9.76 years, and a mean hemoglobin A1c of $7.99 \%$. When asked to rate factors most important in food selection, the highest mean responses were taste (3.97 out of 5) and cost (score of 3.94 out of 5). Barriers that the majority

Todd R. Marcy $(\varangle) \cdot$ Mark L. Britton · Don Harrison Department of Pharmacy, Clinical and Administrative Sciences, University of Oklahoma College of Pharmacy, 1110 N Stonewall, Oklahoma City, OK 73117-1200, USA. Email: Todd-Marcy@ouhsc.edu of respondents agreed or strongly agreed were important included: stress causing over-eating or unhealthy food choices, difficulty resisting the temptation to eat unhealthy food, and healthy food being too expensive. The Cronbach's Alpha for the subscales of food selection, importance of life challenges, and barrier were 0.673, 0.853, and 0.786, respectively. Conclusions: In a lowincome, urban, predominantly African American and Caucasian diabetic population, cost of healthy food, stress-related inappropriate eating, and the temptation to eat unhealthy food were the most frequently reported barriers to healthy eating. Diabetes education programs serving similar populations should evaluate the presence of these barriers. The survey instrument was a reliable measure of the constructs it purported to measure.

Keywords: barriers; diet; low income population; self-care; type 2 diabetes; urban health

\section{INTRODUCTION}

Type 2 diabetes is a significant and growing problem in the United States. An estimated 23.8 million Americans had diabetes in 2007, and the prevalence is growing. ${ }^{1}$ There will be an estimated 
three-fold increase in diabetes prevalence by $2050 .^{2}$ Low-income populations have a higher prevalence of diabetes than higher income populations. ${ }^{3-5}$ Attainment of optimal glycemic control, as assessed by hemoglobin A1c (A1c) values, is established to prevent microvascular complications of diabetes. , $^{6}$ Optimal glycemic control may contribute toward a reduction in macrovascular complications, although pharmacologic-based interventions to aggressively normalize glycemia have not been identified to have a macrovascular benefit. ${ }^{8-10}$ Achievement of blood pressure, lipid, and weight goals is associated with reduced vascular endpoints, especially in patients with type 2 diabetes. ${ }^{11-13}$

Individualized medical nutrition therapy is a component of the optimal care for all patients with type 2 diabetes. ${ }^{13}$ Failure to implement such strategies will increase pharmacologic requirements or result in suboptimal glycemic control. Savoca and colleagues reported that food habits accounted for $51.5 \%$ of the total variance in A1c values in a diabetic population comprised predominantly of low-income African American and Caucasian patients. ${ }^{14}$

Intuitively, patients who adhere to dietary recommendations can expect larger reductions in A1c (up to 1\%) than patients who do not. ${ }^{15}$ However, health professionals and patients note adherence to diet is a significant problem in diabetes management. ${ }^{16,17}$ For example, in a sample of 334 patients at high cardiovascular risk, $63.5 \%$ did not adhere to any diet regimen. ${ }^{18}$ Patients with less apparent barriers tend to adhere to diet and lifestyle modifications more frequently. ${ }^{19}$ Identification of barriers to appropriate eating is a key step in assessment of diabetic patients.

Dietary barriers in diabetic patients have been identified in the literature, primarily through focus group sessions. Barriers often vary based on demographics as well as among patients with the same demographic background. ${ }^{20}$ In focus group sessions of predominantly African American or Caucasian populations, the following barriers were commonly identified: perceived cost of healthy eating, small portion sizes, family support issues, and lifestyle issues. ${ }^{16}$ However, in a focus group of a predominantly Caucasian, rural population, different barriers were identified. ${ }^{17}$ These included: lack of knowledge of a specific diet plan, lack of understanding of their plan of care, and feelings of helplessness/frustration from poor glycemic control despite adherence.

Schlundt and colleagues used structured interviews of diabetic patients to identify clusters of problem situations in regard to adherence to an appropriate diabetic diet. These areas included negative emotions, resisting temptation, eating out, feeling deprived, time pressure, tempted to relapse, meal planning, competing priorities, social events, family support, food refusal, and friends' support. ${ }^{21}$

There are limited data regarding the most common barriers in a low-income, urban, heterogeneous ethnic population. A validated instrument to identify barriers to dietary adherence in these patients is lacking. This validated instrument will be vital for health professionals in identifying barriers to dietary adherence. The objective of this study was to identify barriers to appropriate dietary behavior in an urban, low-income population of patients with type 2 diabetes and to examine a new instrument in the identification of these barriers to appropriate dietary behavior in this population.

\section{METHODS}

\section{Sample/Setting}

Ninety-eight subjects with type 2 diabetes mellitus were recruited and consented to 
complete a survey describing barriers to appropriate eating. Recruitment occurred during visits with their primary-care physician or in a pharmacist/dietitian service providing education and management in a focused diabetes service in the same building. The physician practice is an independent group physician practice administered on an academic health sciences center campus. Men and women with type 2 diabetes, age 18 years or older, who were receiving either Oklahoma Medicaid or were without insurance, and were cared for within the study facility were eligible to participate. Patients with cognitive deficits rendering them incapable of communicating answers were excluded.

\section{Survey Development}

A process to create and validate a new instrument was implemented. The survey instrument was developed by three certified diabetes educators (two pharmacists and one dietitian), one pharmacy administration researcher, one clinical psychologist, and two family practice physicians. The survey instrument was then administered to a test sample of 10 patients. Modifications to improve clarity and readability were subsequently made.

The survey focused on demographic information and three primary question subscales. Respondents answered questions using a five-point Likert scale. The food selection subscale assessed the importance of food content, time, and cost in selecting foods. The importance subscale asked respondents to rate the importance of 10 different common problems in their life, including diabetes. The barrier subscale assessed the factors making dietary adherence difficult. Barrier items were based on Schlundt's taxonomy for obstacles to dietary adherence in patients with diabetes. ${ }^{21}$
The following were specifically assessed in the context of barriers to appropriating eating: perception of food knowledge, food cost, and desire to adhere to an appropriate diet. The Flesch-Kincade reading level for the survey instrument was 5.7. The project was approved by the institutional review board of the participating center.

\section{Survey Administration}

Medical records were screened prior to the clinic visit to identify patients meeting inclusion criteria. Consenting participants were asked to complete the survey either before or after their clinic appointment. Study staff were available to assist the patients in survey completion.

\section{Statistical Analysis}

Descriptive statistics were performed on all survey items. Respondents were categorized into one of two groups based on responses to the various barrier subscale items. One response group consisted of respondents that indicated an agreement or strong agreement to a barrier subscale item. The second group consisted of respondents that indicated a disagreement or strong disagreement to the same barrier subscale item. The mean responses of the two groups of respondents were compared via independent measures t-tests for each survey item in order to assess potential significant differences. The mean survey item responses served as the dependent variables. Group membership (based on responses to the barriers of care subscale response) served as the independent variable. Data analysis was conducted using SPSS for Windows version 14.0 (SPSS Inc., Chicago, IL, USA). The a priori alpha was set at $P \leq 0.05$. 


\section{RESULTS}

\section{Demographics}

Ninety-eight patients completed at least 50\% of the survey. The sample was predominantly Caucasian (43.9\%) and African American (42.9\%). Women comprised nearly $80 \%$ of the sample. The mean age was 51.98 years and mean duration of diabetes was 9.76 years. The mean A1c was 7.99\% (most recent A1c prior to screening). Table 1 lists the demographic information.

\section{Survey Instrument Reliability}

Survey instrument reliability was assessed via the evaluation of subscale reliability. This was accomplished via the calculation of a Cronbach's Alpha for each of the three subscales discussed in this research. The Cronbach's Alpha for the food selection subscale was 0.673 . The Cronbach's Alpha for the importance subscale was 0.853 . The barrier subscale had a Cronbach's Alpha of 0.786. Overall, it appears that the survey instrument was indeed a reliable measure of the various constructs it purported to measure.

\section{Patient Self-assessment of Appropriate Eating Knowledge}

When asked their agreement with the statement, "You have good knowledge about how people with diabetes should eat," the average response was 3.83 (1=strongly disagree, 2=disagree, $3=$ neutral, 4=agree, 5=strongly agree).

Approximately $69 \%$ agreed or strongly agreed with this question and $10.4 \%$ disagreed or strongly disagreed. Most patients agreed or strongly agreed that food selection (93.8\%) and appropriate portion sizes (88.7\%) are important for their overall health.

Table 1. Patient demographics $(n=98)$.

\begin{tabular}{ll}
\hline Demographic & \\
\hline Ethnicity, \% $(n)$ & $42.9(42)$ \\
African American & $2.0(2)$ \\
American Indian & $43.9(43)$ \\
Caucasian & $5.1(5)$ \\
Hispanic & $6.1(6)$ \\
Other/multi-ethnic & \\
Gender & $20.4(20)$ \\
Male & $79.6(78)$ \\
Female & \\
Age, years (standard deviation) & $51.98(11.8)$ \\
Mean & \\
Hemoglobin Alc, \% (standard deviation) & $7.99(2.2)$ \\
Mean & \\
Education, \% (n) & $28.6(28)$ \\
Some high school & $37.8(37)$ \\
High school graduate/GED & $16.3(16)$ \\
Some college & $2.0(2)$ \\
College graduate & $3.1(3)$ \\
Graduate degree & $12.2(12)$ \\
\hline
\end{tabular}

$\mathrm{GED}=$ general educational development. 


\section{Food Selection Subscale}

Subjects were asked to report the individual importance of taste, carbohydrate content, fat content, protein content, cooking time, and cost when choosing what food to eat (Table 2). The highest mean scores were for taste (3.97) and cost (3.94). For these categories nearly 70\% reported the items as being very or extremely important when choosing food. Fat content had the lowest mean (3.36) and the lowest percent answering as very or extremely important (45\%).

\section{Importance Subscale}

When subjects were asked to rate the importance of 10 problems they may face, $66.32 \%$ of patients rated the problem of diabetes as either very important or extremely important (Table $3)$. Health problems besides diabetes had both the highest mean score and the greatest percentage of patients reporting it as either very or extremely important. Other problems with greater than $50 \%$ responding as very or extremely important included: difficulty paying for food, other money problems, problems

Table 2. Patient response to food selection subscale.*

\begin{tabular}{|c|c|c|c|c|}
\hline Category & Mean $(n) \dagger$ & SD & Answered, \% (n) & \\
\hline & & & $1-2$ & $4-5$ \\
\hline Taste & $3.97(92)$ & 0.988 & $6.52(6)$ & $69.57(64)$ \\
\hline Cost & $3.94(97)$ & 1.197 & $14.43(14)$ & $70.10(68)$ \\
\hline Protein & $3.73(97)$ & 1.056 & $12.37(12)$ & $61.86(60)$ \\
\hline Carbohydrate & $3.57(92)$ & 1.170 & $21.74(20)$ & $54.35(50)$ \\
\hline Cooking time & 3.57 (97) & 1.263 & $18.56(18)$ & $52.58(51)$ \\
\hline Fat & $3.36(97)$ & 1.252 & $25.77(25)$ & $46.39(45)$ \\
\hline
\end{tabular}

*Responses to the question "How important is each of the following when you choose what food to eat?".

$\dagger 1=$ not important; $2=$ somewhat important; 3 =important; $4=$ very important; $5=$ extremely important.

Table 3. Patient response to importance subscale.*

\begin{tabular}{lllll}
\hline Category & Mean $(n) \dagger$ & SD & \multicolumn{2}{l}{ Answered, \% $(n)$} \\
\hline & & & $1-2$ & $4-5$ \\
\cline { 5 - 5 } Stress at work & $2.73(83)$ & 1.624 & $44.58(37)$ & $38.55(32)$ \\
Unemployment & $2.77(79)$ & 1.625 & $46.84(37)$ & $36.71(29)$ \\
Health problems besides diabetes & $4.17(96)$ & 1.185 & $10.42(10)$ & $80.21(77)$ \\
Difficulty paying for food & $3.69(94)$ & 1.384 & $19.15(18)$ & $58.51(55)$ \\
Other money problems & $3.85(92)$ & 1.366 & $16.30(15)$ & $64.13(59)$ \\
Problems with side effects from medicine & $3.38(94)$ & 1.482 & $27.66(26)$ & $51.06(48)$ \\
Family stress & $3.76(92)$ & 1.440 & $21.74(20)$ & $65.22(60)$ \\
Emotional difficulties & $3.69(93)$ & 1.351 & $20.43(19)$ & $59.14(55)$ \\
Legal problems & $2.55(94)$ & 1.604 & $52.13(49)$ & $30.85(29)$ \\
Problem of diabetes & $4.03(95)$ & 1.180 & $10.53(10)$ & $66.32(63)$ \\
\hline
\end{tabular}

*Responses to the question "Rate the importance of the following problems that you may experience in your life."

$\dagger 1=$ not important; 2 =somewhat important; 3 =important; $4=$ very important; $5=$ extremely important. 
with medication side effects, family stress, and emotional difficulties.

\section{Barrier Subscale}

There were four barriers to appropriate eating in which $50 \%$ or more of the subjects agreed or strongly agreed (Table 4). These barriers included: stress causing over-eating $(59.38 \%$, mean 3.52, standard deviation 1.314), stress causing unhealthy food choices $(53.76 \%$, mean
3.29, standard deviation 1.441), difficulty resisting temptation to eat unhealthy food (54.64\%, mean 3.44, standard deviation 1.330 ), and healthy food being too expensive (59.57\%, mean 3.51, standard deviation 1.381). There were three other barriers in which $40 \%$ $50 \%$ of the sample agreed or strongly agreed. These included not knowing what foods to choose $(40.21 \%$, mean 3.03, standard deviation 1.295), not knowing how much food to choose (45.83\%, mean 3.15, standard deviation 1.273$)$,

Table 4. Patient responses to the barrier subscale.*

\begin{tabular}{|c|c|c|c|c|}
\hline Category & $\operatorname{Mean}(n) \dagger$ & SD & Answered, \% (n) & \\
\hline & & & $1-2$ & $4-5$ \\
\hline You do not know what food to choose & $3.03(97)$ & 1.295 & $39.18(38)$ & $40.21(39)$ \\
\hline $\begin{array}{l}\text { You do not know how much of each food to } \\
\text { choose }\end{array}$ & $3.15(96)$ & 1.273 & $35.42(34)$ & $45.83(44)$ \\
\hline Stress causes you to eat too much & $3.52(96)$ & 1.314 & $26.04(25)$ & $59.38(57)$ \\
\hline Stress causes you to eat unhealthy food & $3.29(93)$ & 1.441 & $34.41(32)$ & $53.76(50)$ \\
\hline It takes too long to cook healthy food & $2.33(96)$ & 1.185 & $64.58(62)$ & $18.75(18)$ \\
\hline $\begin{array}{l}\text { It is hard to keep saying no to the temptation } \\
\text { eat unhealthy food }\end{array}$ & $3.44(97)$ & 1.330 & $25.77(25)$ & $54.64(53)$ \\
\hline Healthy food costs too much money & $3.51(94)$ & 1.381 & $24.47(23)$ & $59.57(56)$ \\
\hline Someone else cooks your meals & $2.31(93)$ & 1.391 & $59.14(55)$ & $21.51(20)$ \\
\hline $\begin{array}{l}\text { You have family members that make it hard } \\
\text { to eat healthy food }\end{array}$ & $2.50(90)$ & 1.351 & $56.67(51)$ & $28.89(26)$ \\
\hline $\begin{array}{l}\text { You have friends that make it hard to eat } \\
\text { healthy food }\end{array}$ & $2.26(96)$ & 1.242 & $66.67(64)$ & $18.75(18)$ \\
\hline $\begin{array}{l}\text { You have responsibilities that keep you from } \\
\text { choosing the right food }\end{array}$ & $2.26(92)$ & 1.098 & $63.04(58)$ & $13.04(12)$ \\
\hline You feel like you cannot have food you want & $3.18(95)$ & 1.329 & $38.95(37)$ & $44.21(42)$ \\
\hline $\begin{array}{l}\text { Social or work events make it easy to eat } \\
\text { unhealthy food }\end{array}$ & $2.47(88)$ & 1.268 & $57.95(51)$ & $23.86(21)$ \\
\hline $\begin{array}{l}\text { You do not think choosing the right foods is } \\
\text { important }\end{array}$ & $2.77(91)$ & 1.599 & $56.04(51)$ & $37.36(34)$ \\
\hline $\begin{array}{l}\text { You would rather risk the problems you } \\
\text { might get from having high blood sugar than } \\
\text { give up the food you want to eat }\end{array}$ & $2.15(93)$ & 1.293 & $69.89(65)$ & $16.13(15)$ \\
\hline $\begin{array}{l}\text { You believe diabetes is out of your control, } \\
\text { whether you eat right or not }\end{array}$ & $2.59(93)$ & 1.393 & $55.91(52)$ & $26.88(25)$ \\
\hline
\end{tabular}

${ }^{*}$ Responses to the question "Rate each of the following reasons that make it hard in your life to eat food good for patients with diabetes."

$\dagger 1=$ strongly disagree; 2 =disagree; 3 =neutral; $4=$ agree; $5=$ strongly agree. 
and feeling deprived of desired foods (44.21\%, mean 3.18, standard deviation 1.329).

Nearly $65 \%$ of subjects disagreed or strongly disagreed that time to cook healthy food was a barrier to appropriate eating. Most disagreed or strongly disagreed that negative influences of family $(56.67 \%$, mean 2.50 , standard deviation $1.351)$ or friends $(66.67 \%$, mean 2.26 , standard deviation 1.242) were barriers to appropriate eating. Interestingly, nearly $27 \%$ (mean response 2.59 , standard deviation 1.393) agreed or strongly agreed that their diabetes was out of control regardless of the appropriateness of their diet. About 16\% (mean 2.15, standard deviation 1.293) agreed or strongly agreed that they would rather risk complications of diabetes than give up the food they want to eat.

\section{Analysis of Barrier Subscale}

Responses of agreement or strong agreement that healthy food costs too much money were associated with agreement or strong agreement in six other barrier categories. Respondents who agreed or strongly agreed that healthy food costs too much money had a mean response of 3.39 to the statement "you do not know what foods to choose" compared with a response of 2.30 for patients who disagreed or strongly disagreed that healthy food costs too much money $(P=0.001)$. This implies that respondents with greater agreement that healthy food costs too much money were more uncertain about the appropriate food choices.

Respondents who were in agreement or strong agreement (compared with disagreement or strong disagreement) with the statement "healthy food costs too much money" had significantly different mean responses regarding "it takes too long to cook healthy food" (2.54 vs. $1.83, P=0.021)$, "it is hard to keep saying no to the temptation to eat unhealthy food"
(3.68 vs. 2.91, $P=0.024)$, "you feel like you cannot have the food you want" (3.47 vs. 2.78, $P=0.044)$, "social or work events make it easy to eat unhealthy food" (2.55 vs. 2.00, $P=0.040$ ), and "you believe diabetes is out of your control, whether you eat right or not" (2.85 vs. 2.13, $P=0.044)$.

Responses of agreement or strong agreement to the preference to risk complications rather than give up the food they want to eat were associated with agreement or strong agreement in five other barrier categories. Respondents who agreed or strongly agreed with the statement "you would rather risk the problems you might get from having high blood sugar than give up the food you want to eat" had a significantly different mean (3.80) to the statement "you do not know what foods to choose" compared with a mean response of 2.83 in patients who disagreed or strongly disagreed that they preferred to risk complications of diabetes $(P=0.009)$. This implies that patients who would prefer to risk problems associated with hyperglycemia were more uncertain about appropriate food choices.

The mean responses of those respondents who were in agreement or strong agreement (compared with disagreement or strong disagreement) with the statement "you would rather risk the problems you might get from having high blood sugar than give up the food you want to eat" were also significantly different with regard to "stress causes you to eat too much" ( 4.13 vs. $3.36, P=0.012$ ), "stress causes you to eat unhealthy food" ( 4.00 vs. $3.08, P=0.022$ ), "it is hard to keep saying no to the temptation to eat unhealthy food" (4.07 vs. 3.20, $P=0.028$ ), and "you feel like you cannot have food you want" (4.00 vs. $2.91, P=0.003$ ).

Responses of agreement or strong agreement that stress results in excessive food intake were associated with agreement or strong agreement in five other barrier categories. 
Respondents indicating that they agreed or strongly agreed that "stress causes you to eat too much" had a significantly different mean response of 4.13 to the statement "stress causes you to eat unhealthy food" compared with a mean response of 1.67 for those who disagreed or strongly disagreed that stress causes them to eat too much $(P<0.001)$. The mean responses of those in agreement or strong agreement (compared with disagreement or strong disagreement) with the statement "stress causes you to eat too much" were also significantly different with regard to the statements "it takes too long to cook healthy food" (2.46 vs. 1.88, $P=0.016)$, "it is hard to keep saying no to the temptation to eat unhealthy food" (3.67 vs. 2.84, $P=0.013)$, "you don't know how much of each food to choose" (3.25 vs. 2.50, $P=0.020$ ), and "you would rather risk the problems you might get from having high blood sugar than give up the food you want to eat" (2.35 vs. $1.67, P=0.010)$. In addition, respondents who agreed or strongly agreed with the statement "stress causes you to eat too much" had a higher mean response to "emotional difficulties are a problem" (4.11 vs. $2.88, P<0.001)$.

\section{DISCUSSION}

Our study identified barriers to appropriate dietary behaviors in a heterogeneous, lowincome, urban population with type 2 diabetes. Despite an average duration of diabetes of 10 years and the acknowledgment that appropriate eating is important to health, cost and taste had the highest mean response to a question about factors influencing food selection. Approximately half of the sample did not identify carbohydrate or fat content as very or extremely important when making food choices. This indicates either a lack of knowledge or a lack of commitment to an appropriate diet. Barriers with the highest mean responses (increasing agreement) were stress causing inappropriate eating (food choices and portions), excessive cost of healthy food, and difficulty resisting the temptation to eat in an unhealthy way.

There was inconsistency in reporting of knowledge of appropriate eating choices. Most patients agreed or strongly agreed that they had good knowledge of appropriate eating. However, over $40 \%$ agreed or strongly agreed that they do not know what food to choose and how much food to consume. It is unclear whether patients have a dynamic perception of dietary understanding based on the framing of the question or if they are inconsistently forthright. There could be discordance between healthcare professional and patient interpretation of "good knowledge."

Nondiabetes health problems, financial problems (including difficulty paying for food), emotional difficulties, family stress, and problems from medication side effects all appear to be relevant in this population and may contribute as barriers to appropriate dietary behavior by means of stress-driven eating. Further, stressrelated over-eating was correlated to higher responses in several barrier categories, including a willingness to risk complications of diabetes as opposed to changing dietary behavior. Stress may reduce the threshold for other barriers to influence eating choices.

Approximately one in three respondents did not identify the problem of diabetes as very or extremely important. This may be a result of the aforementioned stresses this population experiences. Further, more respondents noted that health problems besides diabetes were very or extremely important than indicated as such for the problem of diabetes. These observations raise questions as to whether there are other problems that are perceived as important by this subset, whether the patient 
is satisfied with the management of their health problems, and whether problems are influencing dietary behavior.

The survey revealed that the cost of food is an important determinant of food choice; inadequate money to buy appropriate food is perceived by many in this population as a problem, and it is frequently identified as a barrier to appropriate eating. Cost of food has been identified as a barrier in other groups of diabetic patients. ${ }^{16,20}$ The observation that perceived high cost of healthy food was associated with patient reporting of inadequate understanding of appropriate food choices indicates that emphasis on low-cost appropriate eating may address this barrier in some patients. The association between the barrier of cost of healthy food with barriers less intuitively associated with inadequate financial resources (eg, cooking time, temptation to eat unhealthy food) requires further study. It is possible that perceived inadequate financial resources may amplify the importance of other barriers and thereby reduce adherence to appropriate dietary behavior.

Based on less than $30 \%$ of patients agreeing or strongly agreeing, it appears the following barriers influence this population of diabetic patients less than other barriers: length of time to cook healthy food, food preparation by another person, family or friends that make healthy eating difficult, concurrent responsibilities, social or work events, preference for complications of diabetes over changing eating lifestyle, and feeling that diabetes is out of control regardless of food intake. However, in any individual patient any of these factors may be a significant barrier to an improved diet.

This study has limitations that must be identified and accounted for when interpreting the results presented. First and foremost is that this study involved self-reported data. There is always the risk that subjects could respond in a socially desirable manner and not respond according to their true attitudes or opinions. However, the threat posed by this limitation is somewhat mitigated by the moderately strong reliability estimates on the various subscales employed in this survey instrument. Another limitation was the nonrandomized selection of the subjects for survey administration. Additionally nonresponse bias could also affect these results. Therefore, the ability to generalize these results to a larger population of diabetic patients could possibly be affected. However, the effect of these limitations is offset, somewhat, by the relatively large differences detected on many of the items surveyed. Lastly, this research was conducted in an academic medical center. The care and intensity of service provided by this clinic may somehow affect patient responses and therefore affect the generalizability of these results to a larger population of diabetic patients.

\section{CONCLUSION}

In a low-income, predominantly African American and Caucasian patient sample, reported barriers were diverse. Cost of healthy food, stress causing inappropriate eating (poor food choices and/or excessive portions), and the temptation to eat unhealthy food were the most frequent barriers in which these patients agreed or strongly agreed. Diabetes education and management programs serving similar populations should address these common barriers. Health professionals must become proactive in identifying and addressing these barriers. The instrument created was effective for identification of these barriers in this population. Further studies are needed to evaluate interventions targeting these barriers. 


\section{ACKNOWLEDGMENTS}

The authors acknowledge the contributions of Frank Lawler, MD, Peter Schwiebert, MD, Paige Beach, RD, MS, CDE, Kevin Farmer, PhD, and Angie Steep, PhD for their contributions in the validation process of the survey instrument. There are no conflicts of interest to report and no extramural funds were used for this project. Todd R. Marcy is the guarantor for this article, and takes responsibility for the integrity of the work as a whole.

Open Access. This article is distributed under the terms of the Creative Commons Attribution Noncommercial License which permits any noncommercial use, distribution, and reproduction in any medium, provided the original author(s) and source are credited.

\section{REFERENCES}

1. Centers for Disease Control and Prevention. National diabetes fact sheet: general information and national estimates on diabetes in the United States, 2007. Atlanta, GA: U.S. Department of Health and Human Services, Centers for Disease Control and Prevention, 2008.

2. Honeycutt AA, Boyle JP, Broglio KR, et al. A dynamic Markov model for forecasting diabetes prevalence in the United States through 2050. Health Care Manag Sci. 2003;6:155-164.

3. Annis AM, Caulder MS, Cook ML, Duquette D. Family history, diabetes, and other demographic and risk factors among the participants of the National Health and Nutrition Examination Survey 1999-2002. Prev Chronic Dis [serial online] April 2005. Accessed November 8, 2010. Available from: URL: http://www.cdc.gov/pcd/issues/2005/ apr/04_0131.htm.

4. Rabi DM, Edwards AL, Southern DA, et al. Association of socio-economic status with diabetes prevalence and utilization of diabetes care services. BMC Health Serv Res. 2006;6:124-130.

5. Connolly V, Unwin N, Sherriff P, Bilous R, Kelly W. Diabetes prevalence and socioeconomic status: a population based study showing increased prevalence of type 2 diabetes mellitus in deprived areas. J Epidemiol Community Health. 2000;54:173-177.

6. UK Prospective Diabetes Group. Intensive bloodglucose control with sulphonylureas or insulin compared with conventional treatment and risk of complications in patients with type 2 diabetes (UKPDS 33). Lancet. 1998;852:837-853.

7. UK Prospective Diabetes Study Group. Effect of intensive blood-glucose control with metformin on complications in overweight patients with type 2 diabetes (UKPDS 34). Lancet. 1998;352:854-865.

8. Stratton IM, Adler AI, Neil HA, et al. Association of glycaemia with macrovasacular and microvascular complications of type 2 diabetes (UKPDS 35): prospective observational study. BMJ. 2000;321:405-412.

9. Selvin E, Marinopoulos S, Berkenblit G, et al. Meta-analysis: glycosylated hemoglobin and cardiovascular disease in diabetes mellitus. Ann Intern Med. 2004;141:421-431.

10. The Action to Control Cardiovascular Risk in Diabetes (ACCORD) Study Group. Effects of intensive glucose lowering in type 2 diabetes. N Engl J Med. 2008;358:2545-2559.

11. Chobanian AV, Bakris GL, Black HR, et al. The Seventh Report of the Joint National Committee on Prevention, Detection, Evaluation, and Treatment of High Blood Pressure: the JNC 7 report. JAMA. 2003;289:2560-2572.

12. Baigent C, Keech A, Kearney PM, et al. Efficacy and safety of cholesterol-lowering treatment: prospective meta-analysis of data from 90,056 participants in 14 randomised trials of statins. Lancet. 2005;366:1267-1278.

13. American Diabetes Association. Standards of Medical Care in Diabetes - 2010. Diabetes Care. 2010;33(suppl. 1):S11-S61.

14. Savoca MR, Miller CK, Ludwig DA. Food habits are related to glycemic control among people with type 2 diabetes mellitus. J Am Diet Assoc. 2004;104:560566.

15. Delahanty LM, Halford BN. The role of diet behaviors in achieving improved glycemic control in intensively treated patients in the diabetes control and complications trial. Diabetes Care. 1993;16:1453-1458. 
16. Vijan S, Stuart NS, Fitzgerald JT, et al. Barriers to following dietary recommendations in type 2 diabetes. Diabet Med. 2005;22:32-38.

17. Nagelkerk J, Reick K, Meengs L. Perceived barriers and effective strategies to diabetes selfmanagement. J Adv Nurs. 2006;54:151-158.

18. Serour M, Alqhenaei H, Al-Saaqabi S, Mustafa AR, Ben-Nakhi A. Cultural factors and patients' adherence to lifestyle measures. Br J Gen Pract. 2007; $57: 291-295$.

19. Aljasem LI, Peyrot M, Wisso L, Rubin RR. The impact of barriers and self-efficacy on self-care behaviors in type 2 diabetes. Diabetes Educ. 2001:27:393-404.

20. Glanz K, Basil M, Maibach E, Goldberg E, Snyder D. Why Americans eat what they do: taste, nutrition, cost, convenience, and weight control concerns as influences on food consumption. J Am Diet Assoc. 1998;98:1118-1126.

21. Schlundt DG, Hargreaves MK, Buchowski MS. The eating behavior patterns questionnaire predicts dietary fat intake in African American women. J Am Diet Assoc. 2003;103:338-345. 